\title{
Tracing Technology Diffusion of Social Media with Culturally Localized User Experience Approach
}

\author{
Huatong Sun \\ Interdisciplinary Arts \& Sciences, University of Washington Tacoma \\ Tacoma, WA 98402, U.S.A \\ huatongs@gmail.com
}

\begin{abstract}
This paper examines two recent technology diffusion cases of social media in a global context, Facebook Japan and Sina Weibo. By tracing the local development of two social media technologies and probing into the deeper issues behind their peculiar use patterns, it presents a new framework-Culturally Localized User Experience (CLUE) for culturally sensitive design and argues the integration of action and meaning in design is key to the success of global social media.
\end{abstract}

Keywords: social media, culturally localized user experience, culturally sensitive design, postcolonial, SNS, microblog, Facebook, Weibo.

\section{Introduction}

For the past decade, social media technologies have acclaimed global successes and altered local cultures. However, the overt instrumentality of a social media technology is often lauded over its implicit values and sociocultural meanings during local uses, and people tend to apply an overly techno-utopian view to interpret the changes it has caused. This type of naïve responses contests and exemplifies a common problem in cross-cultural technology design, the disconnect of action and meaning [19]. As a result, the designed technology is usable, but local users do not relate to it or even resist it.

Cross-cultural studies indicate that those local social media use practices usually develop different communication patterns while responding to local cultural and rhetorical traditions and then take on different use trajectories in various locales $[4,10$, $18,19]$. As Ito [9] suggests, we should regard those local uses "as a heterogeneous set of pathways through diverse sociotechnical ecologies" rather than "a single trajectory toward a universal good" (p. 6). However, those studies tend to focus on comparing and contrasting local differences without linking them to the impact of globalization and its accompanying power relationships. Indeed various local uses of social media technologies are profoundly complicated by the issues of value, identity, power, and hegemony in the postcolonial conditions [8].

In this paper I examine two recent technology diffusion cases of social media in a global context, Facebook Japan and Sina Weibo. By tracing the local development of 
two social media technologies, i.e., Social Network Service (SNS) and microblogging, and probing into the deeper issues behind their peculiar use patterns, I present a new framework-Culturally Localized User Experience (CLUE) [19] for culturally sensitive design and argue the integration of action and meaning in design is key to the success of global social media.

\section{Local Development of Two Social Media Technologies}

Originating from the U.S., social media technologies such as Facebook (SNS) and Twitter (microblogging) have been diffusing rapidly across the globe through themselves and through their copycats. The diffusion processes and the cross-cultural use patterns generated provide a lot of food for thoughts for cross-cultural design practices.

In the first case, the U.S.-based Facebook had a difficult five-year battle to take the Japanese market. As the Facebook has rapidly risen as the world's SNS website with a billion users [20], many local SNS websites were kicked out of the game [3]. However, Facebook might have never expected that it could take so long to win Japanese users. Why were Japanese users reluctant to accept Facebook, which has garnered one victory after another in other places throughout the world rapidly?

Facebook asks its users to use real names and photos for profiles. This distinctive feature that has made Facebook a huge success in American culture where it originated, conflicts with Japanese Internet culture. Japanese users prefer to use pseudonyms to interact with each other either for bonding with old friends or for bridging for new connections, with a different conceptualization of privacy. Over $75 \%$ of Japanese social media users chose to use pseudonyms around the time of 2009 [15], as supported by the top Japanese SNS website Mixi at that time. Consequently Facebook's penetration rate had been stagnating at $3 \%$ for a long time until fall 2011, long after a Japanese version was released in 2008 and after a Facebook office was opened in Tokyo in September 2010.

Facing such strong resistance from local users, the global IT giant determined to conquer this strategically important online market with bold localization initiatives while sticking to their real-name policy. Efforts include promoting the site with missions game, designing a customized mobile interface, allowing users to syndicate Facebook posts on Mixi, and introducing a job search application for college students. It eventually acclaimed victory in September 2012 after morphing into a professional networking site for Japanese users [5], similar to LinkedIn, where local users are more comfortable to use their real names for professional networking.

In the second case, a Chinese copycat of Twitter, Sina Weibo, outperforms Twitter behind the great Firewall of China. Sina Weibo was launched in August 2009 to fill the void after Twitter was blocked by the Chinese government since the Ürümqi riots that July. Here "Weibo" is the Chinese word for "microblogging". With more than 500 million registered users and 46.2 million daily active users as of February 2013, it is ranked as the No. 2 active social network in China $[11,14]$. 
Starting as a copycat, Sina Weibo has adopted many successful features from American SNS websites such as Facebook, Foursquare, Pinterest, and many others. But what really distinguishes Sina Weibo from many other Chinese copycats and makes it a big success should be contributed to its own distinctive features originating from Chinese Internet culture, such as rich media, threaded comment, private chat, microgroup, microevent, and so on. As a matter of fact, it would be highly doubtful whether Twitter would succeed similarly on the Chinese market if not banned.

For example, it is interesting to see how different discursive relationships are built on Sina Weibo and Twitter by comparing how a response and the original author of a reposted message are presented differently on the interfaces of the two. The response to a Weibo message is set up as a threaded comment, under a lower level from the original Weibo, similar to an original post and its responses on a bulletin board system-bulletin board system has been popular in China. In contrast, all the responses on the Twitter are set up on the same level as the original one. In the case of original author of a reposted message, Twitter displays both the reposted message (i.e., retweet) and its original author in the timeline with a fine print noted as "retweeted by" a person one follows. In comparison, what is displayed in the timeline of Sina Weibo is still the user name of the person one follows, and the reposted message is presented as a sublevel message.

In both cases, there is a clear hierarchy of messages in the timeline of Sina Weibo and therefore a hierarchical discursive relationship forms. However, there is not such implied power relationship suggested on the interface of Twitter, and all the messages are treated as an equal contribution in a conversation. Such a difference could be explained with the difference between a collectivist culture like China and an individualist culture like the U.S.: In a collectivist culture, the originality of a microblog message is not necessarily noted in a collaborative discourse.

\section{Integrating Action and Meaning with CLUE}

These two cases above illustrate how important it is to integrate action and meaning in cross-cultural social media design. One common problem in cross-cultural design is the disconnect between action and meaning: Concrete user activities are frequently missing in design practices, and usually only static, out-of-context meanings are transferred through design. As a result, the designed technology is usable, but not meaningful to local users.

To tackle this problem, I developed a design philosophy and methodology Culturally Localized User Experience (CLUE) to create culturally sensitive design for local users [19]. In the CLUE framework, I regard local culture as the dynamic nexus of contextual interactions that manifests numerous articulations of practices and meanings, and advocate a holistic, integrated vision of user experience that takes user experience as both situated action and constructed meaning. Therefore, the CLUE methodology proposes a dialogical, cyclical design process to integrate action and meaning in order to make a technology both usable and meaningful to local users. 
This dialogical, cyclical design process is important and valuable at this stage of glocalization. Glocalization is the third stage of contemporary international communication process after cultural imperialism and globalization [12]. Cultural imperialism is the time when one-way information flow and intentional cultural domination took place. Globalization stresses cultural synchronization and world homogenization and fails to see the dialogicality and complexity of contemporary international cultural relations. Thereby it implies a technology determinist view about development, ignoring local conditions and knowledge [17]. Glocalization recognizes the tension between the global and the local, encourages the interaction of both, and thus theoretically captures "receding center-periphery international arrangements and emerging decentralized, fragmented, and multifaceted patterns" [12, pp. 39-40].

Clearly the Facebook Japan case shows cultural imperialism is still pervasive everywhere, including in an affluent country with self-sufficient Internet culture such as Japan. With the mindset of cultural imperialism, static meanings out of context, influenced by a transmission model of communication [16], are often transferred in crosscultural design, neglecting local cultural preferences and use habits [19]. Meanwhile, the simplistic transmission process and the static meanings transferred are intensified in the postcolonial conditions as both the cultural hybridity and the accompanying power relationships are "unavoidable" [13]. The singularity promoted in the Facebook Japan case - only one social networking mode is honored in this global village-is more alarming and disturbing than similar cases occurring in other developing countries: A local social network service is doomed before this global juggernaut no matter how hard local users fight. It also conflicts with the design goal of "pluralism" we would like to advocate in HCI research and design practices [1, 2, 7]. As Bardzell [1] warns, this type of singularity often "quietly and usually unintentionally imposeswithout transparent or rational justification-Western technological norms and practices" (p. 1305).

On the other hand, the Sina Weibo case suggests the potential of a dialogic design cycle could bring to culturally sensitive design even for a technology with a humble start, i.e., a copycat. It illustrates how new meanings are generated in a technology localization process and how the same technology could bring up different discursive relationships with different interface features. I call the local instances of a globallydiffusing-technology as local uptakes, which is part of the glocalization, informed by Freadman's "uptake" from genre theory [6]. Local uptake is a peculiar phenomenon of this era of glocalization. They represent the pulling forces that re-assert local agency against the pushing force of globalizing trends towards homogeneity and synchronization in contemporary postcolonial conditions.

\section{Conclusion}

Integrating action and meaning is key to the success of global social media. To integrate action and meaning and to design meaningful interactions, the multi-voiced, dialogic approach CLUE argues for dialogic interactions between technology and its surrounding conditions, between action and meaning, between local and global, 
between instrumental and social affordances, and between designers and users. It is dialogism that integrates key concepts of the CLUE framework into a coherent structure, which makes it possible to connect action and meaning in technology design.

\section{References}

1. Bardzell, S.: Feminist HCI: Taking stock and outlining an agenda for design. In: Proc. of CHI 2010: World Conference on Human Factors in Computing Systems, pp. 1301-1310. ACM, New York (2010)

2. Bødker, S.: When second wave HCI meets third wave challenges. In: Proc. of NordiCHI 2006. ACM Press (2006)

3. Cosenza, V.: World map of social networks (December 2012), http://vincos.it/world-map-of-social-networks /

4. Donner, J.: The Rules of Beeping: Exchanging Messages Via Intentional "Missed Calls" on Mobile Phones. Journal of Computer-Mediated Communication 13(1) (2007), http: //jcmc.indiana. edu/vol13/issue1/donner.html

5. Espinosa, J.: Facebook overtakes Japanese social network Mixi in Japan (September 13, 2012),

http: / /www. insidefacebook. com/2012/09/13/facebook-overtakesjapanese-social-network-mixi-in-japan/

6. Freadman, A.: Uptake. In: Coe, R., Lingard, L., Teslenko, T. (eds.) The Rhetoric and Ideology of Genre: Strategies for Stability and Change, pp. 39-53. Hampton Press, Cresskill (2002)

7. Harrison, S., Tatar, D., Sengers, P.: The three paradigms of HCI. In: Ext. Abstracts CHI. ACM Press (2007)

8. Irani, L., Vertesi, J., Dourish, P., Philip, K., Grinter, R.: Postcolonial Computing: A Lens on Design and Development. In: Proceedings of Conference on Human Factors in Computing Systems (CHI 2010), pp. 1311-1320. ACM, New York (2010)

9. Ito, M.: Introduction: Personal, Portable, Pedestrian. In: Ito, M., Okabe, D., Matsuda, M. (eds.) Personal, Portable, Pedestrian Mobile Phones in Japanese Life, pp. 1-16. MIT Press, Cambridge (2006)

10. Ito, M., Okabe, D., Matsuda, M. (eds.): Personal, Portable, Pedestrian Mobile Phones in Japanese Life. MIT Press, Cambridge (2006)

11. Kemp, S.: Social, Digital, Mobile China (January 17, 2013), http://wearesocial.sg/blog/2013/01/social-digital-mobilechina-jan-2013/

12. Kraidy, M.: From Imperialism to Glocalization: A theoretical framework for the Information Age. In: Ebo, B.L. (ed.) Cyberimperialism? Global Relations in the New Electronic Frontier, pp. 27-42. Greenwood Publishing, Westport (2001)

13. Merritt, S., Stolterman, E.: Cultural hybridity and participatory design. In: PDC 2012, Denmark, pp. 73-76 (2012)

14. Ong, J.: China's Sina Weibo grew $73 \%$ in 2012, passing 500 million registered accounts (February 21, 2013), http://thenextweb.com/asia/2013/02/21/chinassina-weibo-grew-73-in-2012-passing-500-million-registeredaccounts/ 
15. Orita, A., Hada, H.: Is that really you?: an approach to assure identity without revealing real-name online. In: Proceedings of the 5th ACM Workshop on Digital Identity Management, pp. 17-20. ACM, New York (2009)

16. Slack, J.: The theory and method of articulation in cultural studies. In: Morley, D., Chen, K.-H. (eds.) Stuart Hall: Critical Dialogues in Cultural Studies, pp. 112-127. Routledge, New York (1996)

17. Slack, J.D., Wise, J.M.: Culture + Technology: A primer. Peter Lang, New York (2005)

18. Sun, H.: Towards a rhetoric of locale: Localizing mobile messaging technology into everyday life. Journal of Technical Writing and Communication 39(2), 245-261 (2009)

19. Sun, H.: Cross-cultural technology design: Creating culture-sensitive technology for local users. Oxford University Press, New York (2012)

20. Zuckerberg, M.: This morning, there are more than one billion people... (October 4, 2012), http: / / www. facebook.com/zuck/posts/10100518568346671 